\title{
Generation of correlated photon pairs in a chalcogenide $\mathbf{A s}_{\mathbf{2}} \mathrm{S}_{\mathbf{3}}$ waveguide
}

\author{
C. Xiong, ${ }^{1,2, a)}$ G. D. Marshall, ${ }^{1,3}$ A. Peruzzo, ${ }^{4}$ M. Lobino, ${ }^{4}$ A. S. Clark, ${ }^{4}$ D.-Y. Choi, ${ }^{1,5}$ \\ S. J. Madden, ${ }^{1,5}$ C. M. Natarajan, ${ }^{6}$ M. G. Tanner, ${ }^{6}$ R. H. Hadfield, ${ }^{6}$ S. N. Dorenbos, ${ }^{7}$ \\ T. Zijlstra, ${ }^{7}$ V. Zwiller, ${ }^{7}$ M. G. Thompson, ${ }^{4}$ J. G. Rarity, ${ }^{4}$ M. J. Steel, ${ }^{1,3}$ \\ B. Luther-Davies, ${ }^{1,5}$ B. J. Eggleton, ${ }^{1,2}$ and J. L. O'Brien ${ }^{4}$ \\ ${ }^{1}$ Centre for Ultrahigh-Bandwidth Devices for Optical Systems (CUDOS), Australia \\ ${ }^{2}$ Institute for Photonics and Optical Science (IPOS), School of Physics, University of Sydney, \\ New South Wales 2006, Australia \\ ${ }^{3}$ MQ Photonics, Department of Physics and Astronomy, Macquarie University, New South Wales 2109, \\ Australia \\ ${ }^{4}$ Centre for Quantum Photonics, H. H. Wills Physics Laboratory, Department of Electrical and Electronic \\ Engineering, University of Bristol, Bristol BS8 1UB, United Kingdom \\ ${ }^{5}$ Laser Physics Centre, Australian National University, Canberra, Australian Capital Territory 0200, \\ Australia \\ ${ }^{6}$ School of Engineering and Physical Sciences, Heriot-Watt University, Edinburgh EH14 4AS, \\ United Kingdom \\ ${ }^{7}$ Kavli Institute for Nanoscience, Delft University of Technology, Lorentzweg 1, 2628 CJ Delft, \\ The Netherlands
}

(Received 4 November 2010; accepted 11 January 2011; published online 31 January 2011)

We demonstrate a $1550 \mathrm{~nm}$ correlated photon-pair source in an integrated glass platform-a chalcogenide $\mathrm{As}_{2} \mathrm{~S}_{3}$ waveguide. A measured pair coincidence rate of $80 \mathrm{~s}^{-1}$ was achieved using 57 $\mathrm{mW}$ of continuous-wave pump. The coincidence to accidental ratio was shown to be limited by spontaneous Raman scattering effects that are expected to be mitigated by using a pulsed pump source. () 2011 American Institute of Physics. [doi:10.1063/1.3549744]

The key to emerging quantum photonic technologies such as quantum communication ${ }^{1}$ and quantum computation ${ }^{2}$ is the availability of bright and low-noise integrated photonpair sources in the $1550 \mathrm{~nm}$ wavelength band. The generation of $1550 \mathrm{~nm}$ correlated photon pairs from photonic-chip platforms, based on parametric downconversion in periodically poled lithium niobate (PPLN) waveguides ${ }^{3}$ and spontaneous four-wave mixing (SFWM) in silicon nanowires, ${ }^{4-6}$ has been widely studied. The PPLN platform requires an external temperature control unit to achieve phase matching and thus is incompatible with integration. The silicon platform is promising because of its mature fabrication technology; however, the two-photon absorption (TPA) induced long lifetime free carriers in silicon result in free-carrier absorption (FCA), which may degrade the source quality. ${ }^{4}$ Thus, there remains a need for $1550 \mathrm{~nm}$ correlated photonpair generation from a platform that supports integration and has no free carriers.

Recent work suggests that the chalcogenide $\mathrm{As}_{2} \mathrm{~S}_{3}$ platform has several potential advantages for integrated $1550 \mathrm{~nm}$ correlated photon-pair sources. ${ }^{7}$ First, $\mathrm{As}_{2} \mathrm{~S}_{3}$ glass exhibits high third-order nonlinearity (comparable to $\mathrm{Si})^{8}$ and fourwave mixing experiments in low-loss $\mathrm{As}_{2} \mathrm{~S}_{3}$ waveguides have demonstrated high parametric gain. ${ }^{9}$ Second, this glass has negligible TPA, making it immune to FCA. ${ }^{8}$ Furthermore, $\mathrm{As}_{2} \mathrm{~S}_{3}$ glass has a low Raman-gain window, which provides a potential means to reduce spontaneous Raman scattering (SpRS) induced noise. ${ }^{7}$ Finally, the glass has the potential to be made into more complex photonic circuits consisting of wavelength division devices and twodimensional/three-dimensional structures. In this letter, we report the observation of $1550 \mathrm{~nm}$ correlated photon-pair generation by SFWM in an integrated chalcogenide $\mathrm{As}_{2} \mathrm{~S}_{3}$

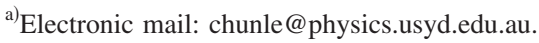

waveguide using a cw pump. By characterizing the dependency of pair coincidence rate on pump power, we show that the measured net pair coincidence rate of $80 \mathrm{~s}^{-1}$ is comparable with that reported in silicon. ${ }^{4,5}$ The coincidence to accidental ratio (CAR), defined as the ratio between the net coincidence rate and the accidental rate, was found to be limited by SpRS, but we expect improvement by using a pulsed pump source so that the pair production is time gated.

Figure 1 shows the experimental setup. The waveguide is $7.1 \mathrm{~cm}$ long. Both facets of the waveguide were infrared antireflection coated to improve coupling and reduce the Fabry-Pérot resonances. Lensed fibers with a mode field diameter of $2.5 \mu \mathrm{m}$ were used to couple to the waveguide. At $1550 \mathrm{~nm}$ the total insertion losses, including propagation and coupling losses, for the fundamental TE and TM modes were measured to be 14.2 and $18.6 \mathrm{~dB}$, respectively. The propagation losses of the TE and TM modes were estimated

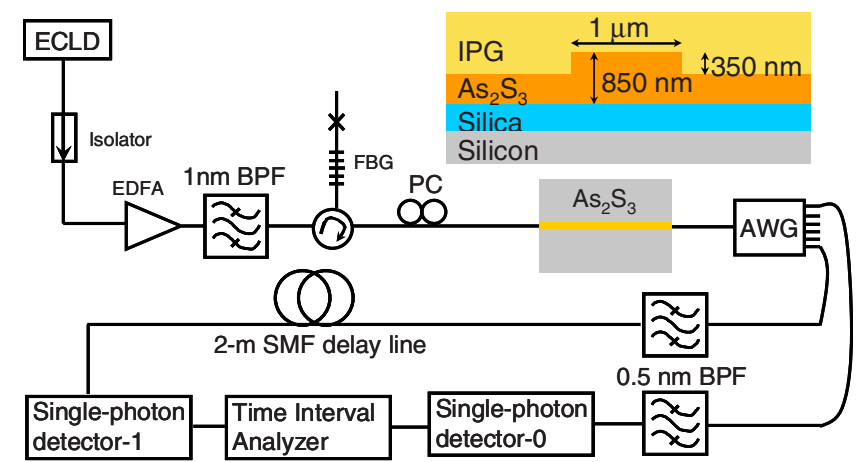

FIG. 1. (Color online) Experimental setup. ECLD: external-cavity laser diode; EDFA: erbium-doped fiber amplifier. A bandpass filter (BPF) with a full width at half maximum (FWHM) of $1 \mathrm{~nm}$, a circulator, and a fiber Bragg grating (FBG) (reflection FWHM of $0.5 \mathrm{~nm}$ ) were employed to reduce noise from the ECLD and EDFA. PC: polarization controller; AWG: arrayed waveguide grating. The inset shows the cross section of the $\mathrm{As}_{2} \mathrm{~S}_{3}$ waveguide. IPG: inorganic polymer glass; SMF: single-mode fiber. 


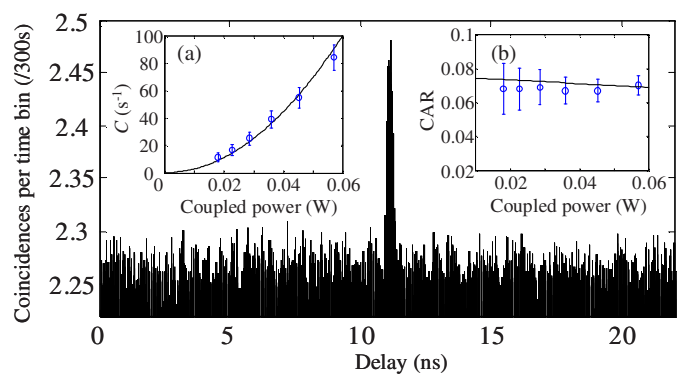

FIG. 2. (Color online) A typical time-bin histogram for coincidence measurements. The insets show (a) the measured net coincidence rate and (b) CAR as a function of coupled pump power. Circles are the measured data. The Poisson error bars are used. In (a), the solid line is a quadratic fit following Eqs. (1); in (b), the solid line is calculated from Eqs. (1).

to be 0.7 and $1.3 \mathrm{~dB} \mathrm{~cm}^{-1}$. The dispersion at $1550 \mathrm{~nm}$ for the TE and TM modes was estimated by a commercial finite-element package (RSOFT FEMSIM) to be -239 and $22 \mathrm{ps} \mathrm{nm}^{-1} \mathrm{~km}^{-1}$, respectively. The nonlinear coefficient was estimated as $\gamma=14 \mathrm{~W}^{-1} \mathrm{~m}^{-1}$ based on the nonlinear refractive index $n_{2}=3 \times 10^{-18} \mathrm{~m}^{2} \mathrm{~W}^{-1}$ and an effective mode area of $0.86 \mu \mathrm{m}^{2}$.

With these parameters and an in-waveguide power of approximately $60 \mathrm{~mW}$ (see Fig. 2), we expect the generation of correlated photon pairs by SFWM spaced equally in frequency about the pump. We refer to the higher (lower) frequency photon as the signal (idler). The output light from the $\mathrm{As}_{2} \mathrm{~S}_{3}$ chip was fed into an AWG, which separated pump and pair photons. The AWG had 40 channels with frequency spacing of $100 \mathrm{GHz}$ and channel FWHM of $50 \mathrm{GHz}$. A tunable BPF with a FWHM of $0.5 \mathrm{~nm}$ was inserted into each of the expected pair-photon channels to further reduce the outof-band noise. The losses of the AWG and BPF were measured to be 6.51 and $6.75 \mathrm{~dB}$ for the two pair-photon channels. After one of the pair photons was optically delayed, they were detected by two fiber-coupled superconducting single-photon detectors (SSPDs) based on NbTiN nanowires. ${ }^{10,11}$ The measured detection efficiencies (at 1550 $\mathrm{nm}$ wavelength and $1000 \mathrm{~s}^{-1}$ dark count rate) were $18 \%$ for detector- 0 and $8 \%$ for detector -1 . The output signals from SSPDs were used as start and stop pulses for a time interval analyzer (TIA).

To produce pairs, we fixed the pump wavelength at $1549.315 \mathrm{~nm}$, corresponding to the center of one AWG channel. We controlled the signal frequency shift $\nu$ from the pump channel using the AWG and BPF with a maximum detuning of $\nu= \pm 1.4 \mathrm{THz}$. All of our experiments used TE polarized pump light as this mode had the lowest propagation loss and provided a SFWM bandwidth that covered the whole of the AWG frequency range. Figure 2 shows a typical time-bin histogram for coincidence measurements when the waveguide-coupled power was $P_{0}=57 \mathrm{~mW}$ (assuming equal division of the coupling losses between input and output) and $\nu=1.4 \mathrm{THz}$. The time bin was $16 \mathrm{ps}$ and the data collection time was $300 \mathrm{~s}$. The sharp peak, located at a delay of $11.1 \mathrm{~ns}$ corresponding to the $2 \mathrm{~m}$ SMF delay line, clearly indicates the generation of correlated photon pairs. The noise floor is due to the accidental events recorded by the TIA and the FWHM of the peak was approximately 200 ps. The idler and signal counting rates at the two detectors were $N_{0}=3.45$ $\times 10^{6} \mathrm{~s}^{-1}$ and $N_{1}=1.34 \times 10^{6} \mathrm{~s}^{-1}$. We analyze the data in terms of the following equations: ${ }^{7,12,13}$

$$
\begin{aligned}
& r=\Delta \nu\left(\gamma P_{0} L_{\mathrm{eff}}\right)^{2} \operatorname{sinc}^{2}\left[\beta_{2}(2 \pi \nu)^{2} L / 2+\gamma P_{0} L\right] \\
& C=\sigma \eta_{\alpha}^{2} \eta^{2} \eta_{0} \eta_{1} r \\
& N_{0}=\sigma \eta \eta_{0}\left(\eta_{\alpha} r+r_{n 0}\right)+d_{0}, \\
& N_{1}=\sigma \eta \eta_{1}\left(\eta_{\alpha} r+r_{n 1}\right)+d_{1}, \\
& A=N_{0} N_{1} t,(\mathrm{cw}) \\
& \mathrm{CAR}=C / A,
\end{aligned}
$$

where $r$ is the rate of photon-pair generation by SFWM, $\Delta \nu$ is the bandwidth of the pair-photon channel, $L$ is the waveguide length and $L_{\text {eff }}=[1-\exp (-\alpha L)] / \alpha$ is the effective waveguide length accounting for the propagation loss $\alpha, \beta_{2}$ $=3.048 \times 10^{-25} \mathrm{~s}^{2} \mathrm{~m}^{-1}$ is the second-order dispersion parameter, $C$ is the net coincidence rate, $\eta_{\alpha}$ is a parameter taking into account the pair-photon loss in the waveguide and can be determined by comparing the measured coincidence rate $C$ and the calculated photon-pair generation rate $r, \eta$ is the waveguide output coupling efficiency, $\eta_{0}=0.04$ and $\eta_{1}$ $=0.017$ are the photon collection efficiencies, including the AWG, the BPF transmission efficiency, and the SSPD quantum efficiency, $r_{n \mathrm{i}}(\mathrm{i}=0,1)$ is the rate of noise-photon generation, $d_{0}=d_{1}=1000 \mathrm{~s}^{-1}$ are the rates of SSPD dark count, $A$ is the accidental rate in the time window of $t$ for the coincidence measurement (i.e., the FWHM of the coincidence peak), and $\sigma=\tau B$ ( $\tau$ is pulse width and $B$ is a pulse repetition rate), is a duty cycle parameter which for cw operation is 1 . Finally, CAR is the coincidence to accidental ratio introduced earlier.

Equations (1) indicate that the net pair coincidence rate should depend quadratically on the coupled pump power. To confirm this we investigated this dependency for $\nu$ $=1.4 \mathrm{THz}$ by varying the pump power and keeping all other conditions unchanged. At each point we obtained a time-bin histogram and extracted the net coincidence rate by subtracting the accidental rate from the coincidence peak. The data presented in the inset (a) of Fig. 2 confirm the expected quadratic dependency.

Pair brightness and correlation are the two standard measurements of the quality of a photon-pair source. The maximum measured pair coincidence rate was about $80 \mathrm{~s}^{-1}$. This is comparable with that reported in silicon ${ }^{4,5}$ and could be further improved with enhanced waveguide coupling enabled by inverse taper coupling sections. ${ }^{4-6}$ The pair correlation is experimentally characterized by the CAR. The inset (b) of Fig. 2 shows the dependency of CAR on pump power. As can be seen, the CAR is low and relatively constant in the operating power range due to the high level of noise. The possible noise sources are the residual pump photons, photons generated in the silica-fiber connecting components, and SpRS in the chalcogenide waveguide itself. The first two possibilities were easily eliminated through tests by using one additional pump rejection FBG and removing the chalcogenide waveguide from the setup. To determine if SpRS in the waveguide itself was the dominant noise, we measured the single count rates as a function of pump power at $\nu$ $=1.4 \mathrm{THz}$. Figure 3 shows the measured and predicted single count rates due to SFWM (solid lines) and SpRS (dotted lines) at different pump powers. ${ }^{7}$ It is clear that most of the single count rate is attributable to SpRS. The count rate 


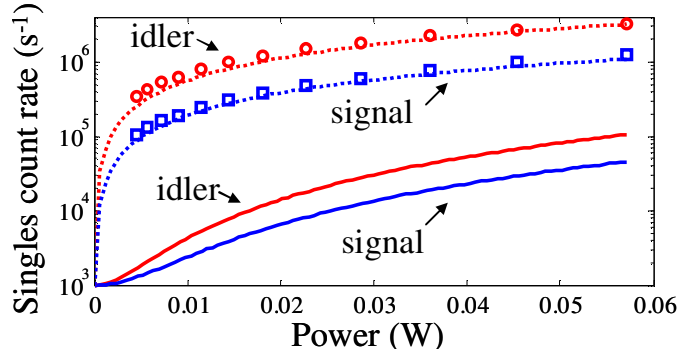

FIG. 3. (Color online) Single count rate as a function of coupled pump power. Circles and squares show the measured idler and signal count rates. Lines show the predicted count rates for idler (red) and signal (blue) from pure SpRS (dotted) and pure SFWM (solid), respectively, using Eqs. (1) and SpRS equations in Ref. 7.

difference between signal and idler channels was due to the different quantum efficiencies of the two detectors and was confirmed by repeating the measurement with the detector positions swapped. It is important to note that the SpRS contribution to the single count rates is almost the same for both the signal and idler channels, a phenomenon expected when the frequency detuning $\nu$ is small. ${ }^{12} \mathrm{We}$ also investigated the dependency of CAR on the frequency detuning $\nu$. We found that the CAR was relatively constant over the range of 0.6$1.4 \mathrm{THz}$. Below $\nu=0.6 \mathrm{THz}$, the CAR dropped quickly as the impact of residual pump photons transmitted by the AWG and BPF becomes significant close to the pump wavelength.

Figure 3 indicates that the noise performance of our source was principally limited by the generation of SpRS noise, a phenomenon expected in glass platforms with a cw pump. ${ }^{13}$ In the future, the use of a pulsed pump laser will provide time-gated pair production and immediately improve the CAR. This can be understood by considering Eqs. (1) in a pulsed pump regime, in which the time window for pair and Raman noise generation is $\tau \ll t$, so $A=\left[\tau \eta \eta_{0}\left(\eta_{\alpha} r+r_{n 0}\right)\right.$ $\left.+d_{0} t\right]\left[\tau \eta \eta_{1}\left(\eta_{\alpha} r+r_{n 1}\right)+d_{1} t\right] B$. Observe that the CAR is independent on $B$, and $\mathrm{CAR}_{\text {pulse }} / \mathrm{CAR}_{\mathrm{cw}}=t / \tau$ if we can neglect the dark count, which is the case for the superconducting detectors. To illustrate this, we plot the predicted CAR as a function of average number of generated pairs per pulse in Fig. 4 (solid line). The calculation is based on Eqs. (1) and all parameters used in our experiment, with $\tau=5 \mathrm{ps}$. It can be seen that the CAR will be 3 at an average pair generation rate of 0.001 per pulse (at $0.31 \mathrm{~W}$ of peak power). Our previous theoretical analysis has shown that the $\mathrm{As}_{2} \mathrm{~S}_{3}$ glass has a low Raman-gain window at a shift of approximately $\nu$ $=7.4 \mathrm{THz}$ for which the CAR is predicted to be much improved. ${ }^{7}$ This experiment, however, did not operate in this regime due to the limited SFWM bandwidth of our existing waveguide device. In future experiments, we plan to lower the dispersion of the waveguide at $1550 \mathrm{~nm}$ by modifying its cross-section size to extend the SFWM bandwidth so that we can produce photons in the $7.4 \mathrm{THz}$ low Raman noise window. ${ }^{7}$ The dotted line in Fig. 4 predicts the CAR in such a dispersion engineered waveguide. The CAR is expected to be 33 at a level of 0.001 generated pairs per pulse. Chalcogenide waveguides are particularly attractive in the pulsed regime as the lack of FCA removes a limit on the peak pump power which is then determined only by the requirement to keep double pair production at an acceptable level (with this

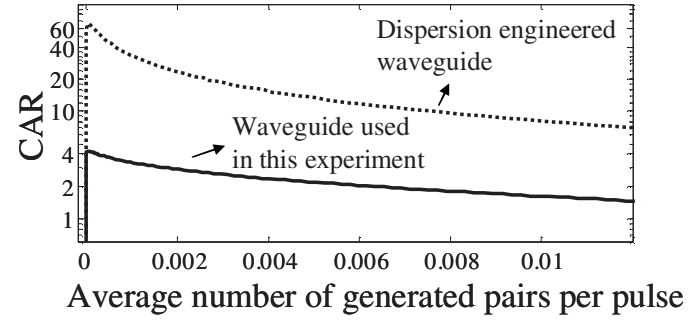

FIG. 4. The predicted CAR as a function of average number of gernerated pairs per pulse in a pulsed pump scheme.

requirement, the peak power is far below the damage threshold of the waveguide ${ }^{8}$ ).

In conclusion, we have demonstrated correlated photonpair generation in the $1550 \mathrm{~nm}$ wavelength band from an integrated chalcogenide $\mathrm{As}_{2} \mathrm{~S}_{3}$ glass waveguide. The measured pair coincidence rate of $80 \mathrm{~s}^{-1}$ is comparable with results in silicon waveguides. The CAR was limited by the SpRS noise in the cw pump scheme. Using a pulsed pump can immediately enhance the CAR to 3 at an average pair generation rate of 0.001 per pulse. Superior waveguide design including dispersion engineering and inverse taper coupling should provide additional improvement. Combined with its suitability for compact nonlinear processing components, ${ }^{8,9}$ we believe that this work opens the way for $\mathrm{As}_{2} \mathrm{~S}_{3}$ and related nonlinear glasses to play a significant role in quantum information processing.

We acknowledge the support of the Australian Research Council Centre of Excellence and Federation Fellowship programs. the Australian Academy of Science's International Science Linkages scheme (G.D.M.), the Royal Society of London University Research Fellowship (R.H.H.), the VIDI funding by FOM (S.N.D., T.Z., and V.Z.), the EU project Q essence, the EU FP7 project QUANTIP, and the Bristol Centre for Nanoscience and Quantum Information. C.M.N., M.G.T., and R.H.H. acknowledge support from EPSRC (U.K.) and assistance in constructing the SSPDs from Dr. S. Nam at NIST, USA.

${ }^{1}$ N. Gisin and R. Thew, Nat. Photonics 1, 165 (2007).

${ }^{2}$ J. L. O'Brien, A. Furusawa, and J. Vučković, Nat. Photonics 3, 687 (2009).

${ }^{3}$ M. Hunault, H. Takesue, O. Tadanaga, Y. Nishida, and M. Asobe, Opt. Lett. 35, 1239 (2010).

${ }^{4}$ J. E. Sharping, K. F. Lee, M. A. Foster, A. C. Turner, B. S. Schmidt, M. Lipson, A. L. Gaeta, and P. Kumar, Opt. Express 14, 12388 (2006).

${ }^{5}$ S. Clemmen, A. Perret, S. K. Selvaraja, W. Bogaerts, D. van Thourhout, R. Baets, Ph. Emplit, and S. Massar, Opt. Lett. 35, 3483 (2010).

${ }^{6}$ K.-I. Harada, H. Takesue, H. Fukuda, T. Tsuchizawa, T. Watanabe, K. Yamada, Y. Tokura, and I. Sei-ichi, IEEE J. Sel. Top. Quantum Electron. 16, 325 (2010).

${ }^{7}$ C. Xiong, L. G. Helt, A. C. Judge, G. D. Marshall, M. J. Steel, J. E. Sipe, and B. J. Eggleton, Opt. Express 18, 16206 (2010).

${ }^{8}$ V. G. Ta'eed, N. J. Baker, L. Fu, K. Finsterbusch, M. R. E. Lamont, D. J. Moss, H. C. Nguyen, B. J. Eggleton, D. Y. Choi, S. Madden, and B. Luther-Davies, Opt. Express 15, 9205 (2007).

${ }^{9}$ M. R. E. Lamont, B. Luther-Davies, D. Y. Choi, S. Madden, X. Gai, and B. J. Eggleton, Opt. Express 16, 20374 (2008).

${ }^{10}$ M. G. Tanner, C. M. Natarajan, V. K. Pottapenjara, J. A. O'Connor, R. J. Warburton, R. H. Hadfield, B. Baek, S. Nam, S. N. Dorenbos, E. Bermúdez Ureña, T. Zijlstra, T. M. Klapwijk, and V. Zwiller, Appl. Phys. Lett. 96, 221109 (2010).

${ }^{11}$ S. N. Dorenbos, E. M. Reiger, U. Perinetti, V. Zwiller, T. Zijlstra, and T. M. Klapwijk, Appl. Phys. Lett. 93, 131101 (2008).

${ }^{12}$ Q. Lin, F. Yaman, and G. P. Agrawal, Phys. Rev. A 75, 023803 (2007).

${ }^{13}$ J. G. Rarity, J. Fulconis, J. Duligall, W. J. Wadsworth, and P. St. J. Russell, Opt. Express 13, 534 (2005). 